Article

\title{
Extracellular Hb Enhances Cardiac Toxicity in Endotoxemic Guinea Pigs: Protective Role of Haptoglobin
}

Jin Hyen Baek ${ }^{1}$, Xiaoyuan Zhang ${ }^{1}$, Matthew C. Williams ${ }^{1}$, Dominik J. Schaer ${ }^{2}$, Paul W. Buehler ${ }^{1}$ and Felice D'Agnillo ${ }^{1, *}$

1 Laboratory of Biochemistry and Vascular Biology, Division of Hematology, Center for Biologics Evaluation and Research, Food and Drug Administration, Bethesda, MD 20892, USA; E-Mails: jin.baek@fda.hhs.gov (J.H.B.); xiaoyuan.zhang@fda.hhs.gov (X.Z.); matthew.williams@fda.hhs.gov (M.C.W.); paul.buehler@fda.hhs.gov (P.W.B.)

2 Division of Internal Medicine, University Hospital, CH-8091 Zurich, Switzerland; E-Mail: dominik.schaer@usz.ch

* Author to whom correspondence should be addressed; E-Mail: felice.dagnillo@fda.hhs.gov; Tel.: +1-301-451-3985; Fax: +1-301-402-2780.

Received: 31 January 2014; in revised form: 18 March 2014 / Accepted: 21 March 2014 / Published: 31 March 2014

\begin{abstract}
Endotoxemia plays a major causative role in the myocardial injury and dysfunction associated with sepsis. Extracellular hemoglobin $(\mathrm{Hb})$ has been shown to enhance the pathophysiology of endotoxemia. In the present study, we examined the myocardial pathophysiology in guinea pigs infused with lipopolysaccharide (LPS), a Gram-negative bacterial endotoxin, and purified $\mathrm{Hb}$. We also examined whether the administration of the $\mathrm{Hb}$ scavenger haptoglobin $(\mathrm{Hp})$ could protect against the effects observed. Here, we show that $\mathrm{Hb}$ infusion following LPS administration, but not either insult alone, increased myocardial iron deposition, heme oxygenase-1 expression, phagocyte activation and infiltration, as well as oxidative DNA damage and apoptosis assessed by 8 -hydroxy-2'-deoxyguanosine $(8-\mathrm{OHdG})$ and terminal deoxynucleotidyl transferase dUTP nick end labeling (TUNEL) immunostaining, respectively. Co-administration of $\mathrm{Hp}$ significantly attenuated the myocardial events induced by the combination of LPS and $\mathrm{Hb}$. These findings may have relevant therapeutic implications for the management of sepsis during concomitant disease or clinical interventions associated with the increased co-exposures to LPS and $\mathrm{Hb}$, such as trauma, surgery or massive blood transfusions.
\end{abstract}


Keywords: hemoglobin; endothelium; endotoxin; haptoglobin; myocardial injury

\section{Introduction}

Myocardial dysfunction and injury are key pathophysiological events in sepsis and septic shock [1,2]. Lipopolysaccharide (LPS), a Gram-negative bacterial endotoxin, is a primary mediator of the cardiovascular dysfunction associated with sepsis or septic shock [3,4]. The mechanisms underlying LPS-mediated cardiovascular dysfunction are multifactorial, but generally implicate the overproduction of cytokines (i.e., tumor necrosis factor-alpha, interleukin 1-beta) and reactive oxygen/nitrogen species as causative processes $[3,4]$.

Several studies have demonstrated that LPS pathophysiology can be exacerbated by extracellular hemoglobin $(\mathrm{Hb})$ [5-7]. In mice, intra-peritoneal injection of LPS combined with intravascular infusion of purified $\mathrm{Hb}$ induced greater mortality compared to $\mathrm{Hb}$ or LPS alone [5]. In rabbits, intravenous administration of chemically cross-linked $\mathrm{Hb}$ and LPS caused significantly greater cardiovascular dysfunction and mortality compared to $\mathrm{Hb}$ or LPS alone [6]. Murine models of infection coupled with a deficiency in heme oxygenase-1 (HO-1), a key enzyme of heme catabolism, suggest that the release of heme following hemolysis increases mortality [8]. In humans, the level of hemolysis occurring during Gram-negative bacteremia may be associated with an increased risk for mortality [9,10]. Plasma $\mathrm{Hb}$ levels in these patients were reported to occur over a range of concentrations from 10 to $40 \mathrm{mg} / \mathrm{dL}$ (equivalent to $\sim 5$ to $25 \mu \mathrm{M}$ as heme) [9,10]. Reduced plasma levels of hemopexin and haptoglobin $(\mathrm{Hp})$ also correlated with an increased risk for mortality in septicemia [11].

$\mathrm{Hb}$ enhancement of LPS pathophysiology may be particularly relevant in Gram-negative sepsis with concomitant trauma, surgery or blood transfusions when there is an increased likelihood for interaction between bacterial cell wall LPS and circulating extracellular $\mathrm{Hb}$. Under such circumstances, it is postulated that the therapeutic management of Hb-LPS synergism may significantly ameliorate clinical outcomes. $\mathrm{Hp}$ is the $\mathrm{Hb}$ scavenger in the plasma of mammals, existing in humans as three primary phenotypes (Hp 1-1, Hp 2-1 and Hp 2-2) within a concentration range of $0.3-1.9 \mathrm{mg} / \mathrm{mL}[12,13]$. These three Hp polymorphisms all contain the same $\mathrm{Hb}$ binding $\beta$ globin $\left(\mathrm{Hp}^{\beta}\right)$, but differ in their $\alpha$ globin $\left(\mathrm{Hp}^{\alpha 1}\right.$ or $\left.\mathrm{Hp}^{\alpha 2}\right)$ composition $[14,15]$. This results in dimeric (Hp 1-1) or multimeric (Hp 2-1, Hp 2-2) forms, as denoted by the number of $\alpha$ globin cysteines involved in disulfide bond formation. Several studies have shown that $\mathrm{Hp}$ prevents $\mathrm{Hb}$ renal excretion and subsequent kidney injury following experimental and clinical hemolysis [16-18]. In the present study, we evaluated the acute myocardial responses of guinea pigs challenged with LPS and Hb with or without the co-administration of purified multimeric human Hp. 


\section{Results}

\subsection{Sequestration of $\mathrm{Hb}$ by Hp Prevents Renal Filtration}

A primary function of plasma $\mathrm{Hp}$ is to prevent renal excretion of $\mathrm{Hb}$ dimers [19]. To validate that the $\mathrm{Hp}$ administration conditions used in the present model effectively bound and maintained the extracellular $\mathrm{Hb}$ within a Hb-Hp complex, we first examined the renal deposition of non-heme iron as a marker of $\mathrm{Hb}$ exposure. Non-heme iron deposition was not observed in surgical control and LPS-infused animals using the Perls diaminobenzidine (DAB) intensification method (Figure 1A,B). However, $\mathrm{Hb}$ and LPS $+\mathrm{Hb}$ administered animals showed non-heme iron accumulation in the tubular epithelium of the renal cortex at $24 \mathrm{~h}$ post exposure. This was visualized as brown staining within hemosiderin granules (Figure 1C-E). Conversely, when LPS $+\mathrm{Hb}$ infusions were combined with concomitant $\mathrm{Hp}$ administration, no renal non-heme iron deposits were visualized (Figure 1F).

Figure 1. Hp prevents renal exposure to $\mathrm{Hb}$. Renal non-heme iron deposition was not detectable in surgical control animals (A) or with LPS alone (B). Animals infused with $\mathrm{Hb}$ alone $(\mathbf{C})$ or LPS $+\mathrm{Hb}(\mathbf{D}, \mathbf{E})$ demonstrated increased iron deposits in the tubular epithelium of the renal cortex (brown staining). The asterisk in Panels $\mathrm{C}$ and $\mathrm{D}$ denote the lumen of a representative tubule containing iron deposits. Administration of $\mathrm{Hp}$ with $\mathrm{Hb}$ following LPS prevented iron deposition (F). Panels A-D and F, 400×; Panel E, 600×. (G) SEC-HPLC chromatographs of plasma samples, purified Hb standard (red trace), and a purified $\mathrm{Hb}-\mathrm{Hp}$ standard (blue trace). (H) Representative images of urine samples $1 \mathrm{~h}$ after dosing. Scale bars $=50 \mu \mathrm{m}$.
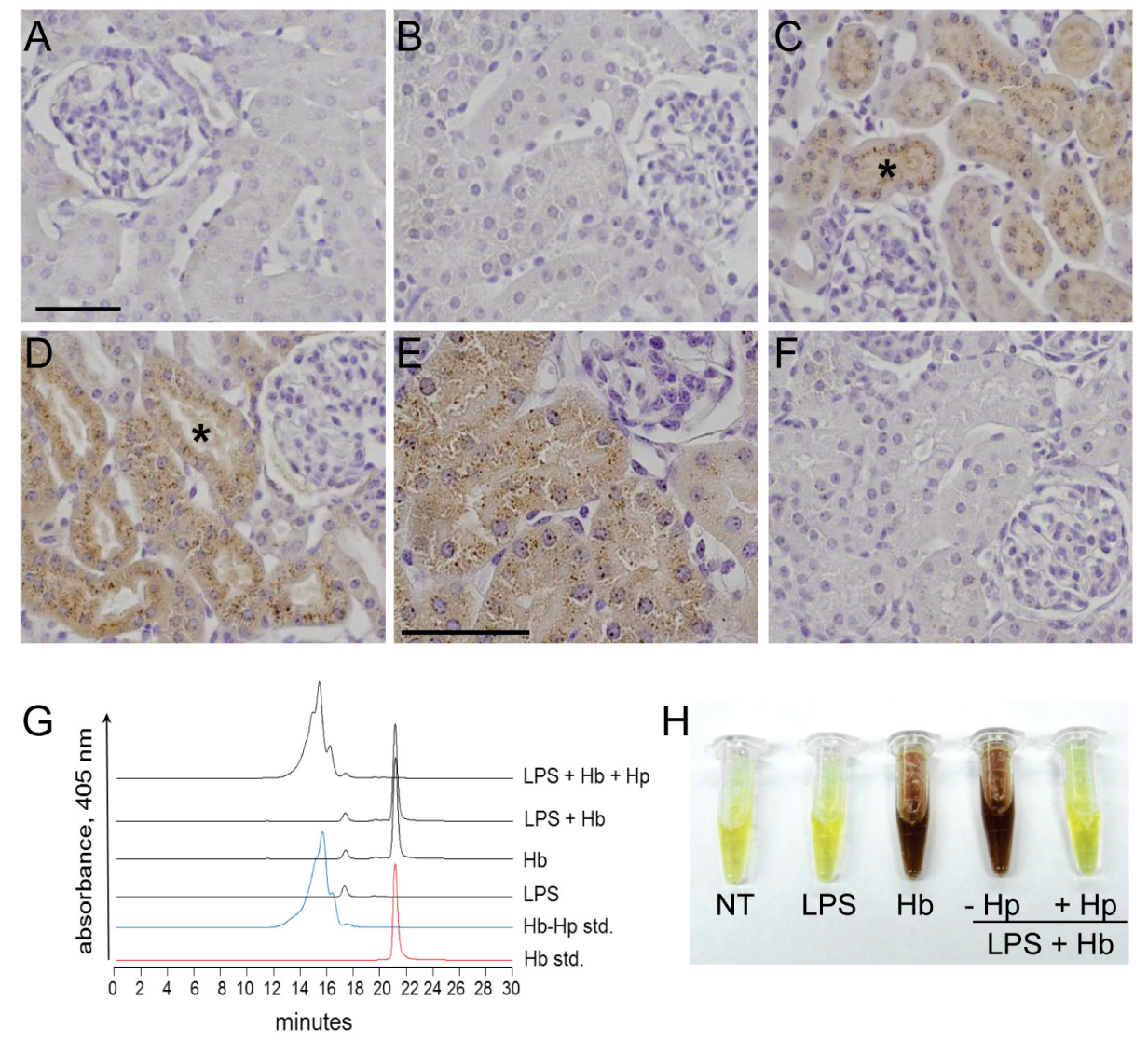

$\mathrm{H}$

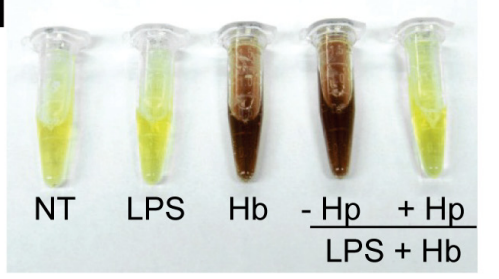


These findings are supported by size exclusion (SEC)-HPLC analysis of plasma samples collected $1 \mathrm{~h}$ after dosing (Figure $1 \mathrm{G}$ ). Representative samples from $\mathrm{Hb}$ and LPS $+\mathrm{Hb}$ administered animals demonstrated a single free $\mathrm{Hb}$ peak at an elution time of $21 \mathrm{~min}$. This was confirmed by the elution time profile of the $\mathrm{Hb}$ standard solution. In the presence of $\mathrm{Hp}, \mathrm{Hb}$ was completely bound and the elution time shifted to a broad band of peaks eluting over 13-17 min. This was also consistent with the elution time profile of the $\mathrm{Hb}-\mathrm{Hp}$ standard. As a final confirmation, $\mathrm{Hp}$ sequestration of $\mathrm{Hb}$ was measured as the extent of hemoglobinuria over the initial hour after dosing (Figure $1 \mathrm{H}$ ). Urinary $\mathrm{Hb}$ accumulation was only observed in $\mathrm{Hb}$ and LPS $+\mathrm{Hb}$ administered animals, but not in surgical controls, LPS or LPS $+\mathrm{Hb}+\mathrm{Hp}$ administered animals. These data confirm that the Hp dose used in this study was effective in scavenging and maintaining all extracellular $\mathrm{Hb}$ within an $\mathrm{Hp}$-bound complex.

\subsection{Hp Prevents Myocardial Iron Deposition and HO-1 Induction by LPS plus Hb}

Non-heme iron deposition was not observed in cardiac sections from surgical control or endotoxemic animals (Figure $2 \mathrm{~A}-\mathrm{C}$ ). Infusion with $\mathrm{Hb}$ alone produced minor, but detectable, iron deposition in focal myocytes (Figure 2D). Animals infused with LPS followed by Hb showed diffuse regions of iron accumulation within myocytes at $24 \mathrm{~h}$ post-Hb infusion (Figure 2E,F). Non-heme iron staining was also observed in endothelial cells and perivascular regions of small and medium-sized cardiac blood vessels (Figure 2G,H). Conversely, the administration of $\mathrm{Hp}$ with $\mathrm{Hb}$ in endotoxemic animals prevented iron deposition in the contractile tissue of the heart and within myocardial blood vessels (Figure 2I). These data suggest that $\mathrm{Hp}$ attenuates extravascular translocation of $\mathrm{Hb}$ and iron deposition within the myocardium of endotoxemic animals.

To further evaluate myocardial exposure to $\mathrm{Hb}$, we examined $\mathrm{HO}-1$ expression by Western blot. No significant changes in HO-1 expression were observed in animals infused with LPS or $\mathrm{Hb}$ alone (Figure 2J). In contrast, animals infused with LPS + Hb showed a six-fold increase in HO-1 expression $24 \mathrm{~h}$ post-infusion compared to surgical controls. Co-administration of $\mathrm{Hp}$ with $\mathrm{Hb}$ prevented the increase in HO-1 expression.

To identify the HO-1-expressing cells, myocardial sections were double labeled for HO-1 and CD163, a marker of monocytes/macrophages. In surgical controls, CD163 ${ }^{+}$perivascular cells that showed no HO-1 reactivity were detectable as flattened and elongated cells around small to medium-sized blood vessels (Figure 3A-C). In $\mathrm{Hb}$ infused animals, CD163 ${ }^{+}$perivascular cells were primarily negative for HO-1, although a few cells with a low level HO-1 reactivity were detectable (Figure 3D-F). In endotoxemic animals, $\mathrm{CD}_{163^{+}}$perivascular cells showed no HO-1 reactivity (Figure 3G-I). In contrast, LPS $+\mathrm{Hb}$ animals showed an increased accumulation of CD163 ${ }^{+} / \mathrm{HO}-1^{+}$ cells around and within small to medium-sized myocardial blood vessels (Figure 3J-L) and in the adventitial regions of large epicardial blood vessels (Figure $3 \mathrm{M}-\mathrm{O}$ ). Hp administration attenuated the induction of HO-1 among CD163 ${ }^{+}$cells in the myocardium (Figure 3P-R) and around large blood vessels (Figure 3S-U). 
Figure 2. Myocardial non-heme iron staining in surgical control animals (A,B), LPS alone (C), $\mathrm{Hb}$ alone (D), LPS $+\mathrm{Hb}(\mathbf{E}-\mathbf{H})$, and LPS $+\mathrm{Hb}+\mathrm{Hp}(\mathbf{I})$. High magnification of the boxed region shows cytoplasmic and nuclear iron staining in myocytes (brown) and surrounding myocytes containing only punctate nuclear iron deposits (F). Arrowheads show iron deposits in the perivascular regions and endothelium of cardiac blood vessels $(\mathrm{G}, \mathrm{H})$ $(600 \times)$. Scale bars $=200 \mu \mathrm{m}(\mathrm{A}$ and $\mathrm{E})$ or $25 \mu \mathrm{m}(\mathrm{B}-\mathrm{D}, \mathrm{F}-\mathrm{I})$. $(\mathbf{J})$ Myocardial HO-1 expression by Western blot. Representative blots for HO-1 and tubulin are shown. Densitometry values as a ratio to tubulin were normalized to non-treated controls (NT) and presented as the means \pm SEM. ${ }^{*} p<0.05 v s$. NT ( $n=4$ animals per group).

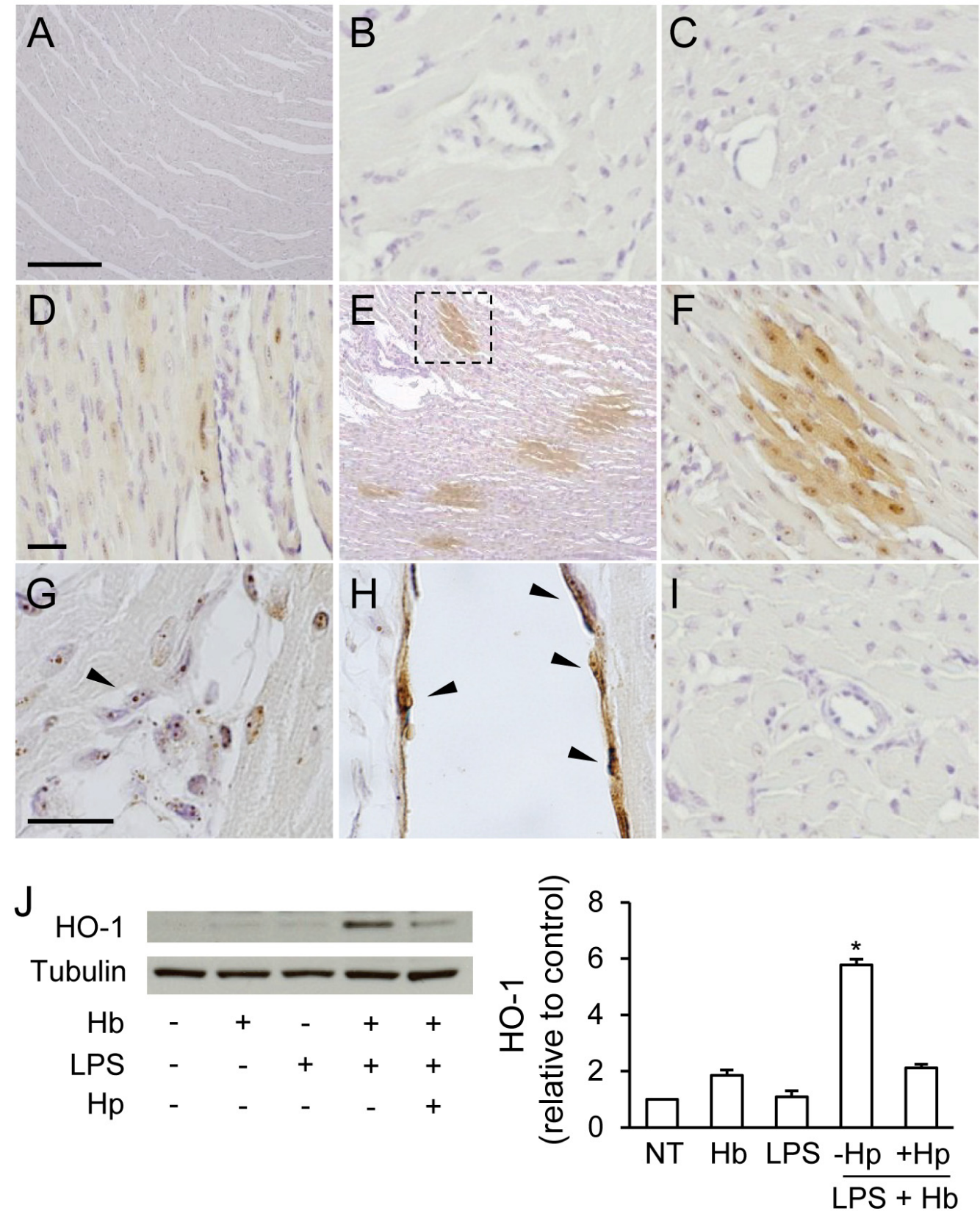


Figure 3. Immunofluorescence analysis of myocardial HO-1 and CD163 expression. Surgical non-treated (NT) control animals showed minimal HO-1 (green) expression in resident $\mathrm{CD}_{163^{+}}$perivascular macrophages (red) (A-C). Sporadic HO-1 expression (white arrow) localized to $\mathrm{CD}_{163}{ }^{+}$cells observed with $\mathrm{Hb}$ alone (D-F). With LPS alone, CD163 infiltrates and perivascular macrophages showed minimal HO-1 expression (white arrowhead) (G-I). Increased HO-1 expression localized to $\mathrm{CD}_{163^{+}}$infiltrates and perivascular macrophages observed with LPS $+\mathrm{Hb}$ (white arrows) (J-L,M-O). Hp reduces HO-1 expression in $\mathrm{CD}_{163^{+}}$infiltrates and perivascular cells $(\mathbf{P}-\mathbf{R}, \mathbf{S}-\mathbf{U})$. Nuclei were counterstained with Hoechst 33342 (blue). Scale bar $=10 \mu \mathrm{m}$.

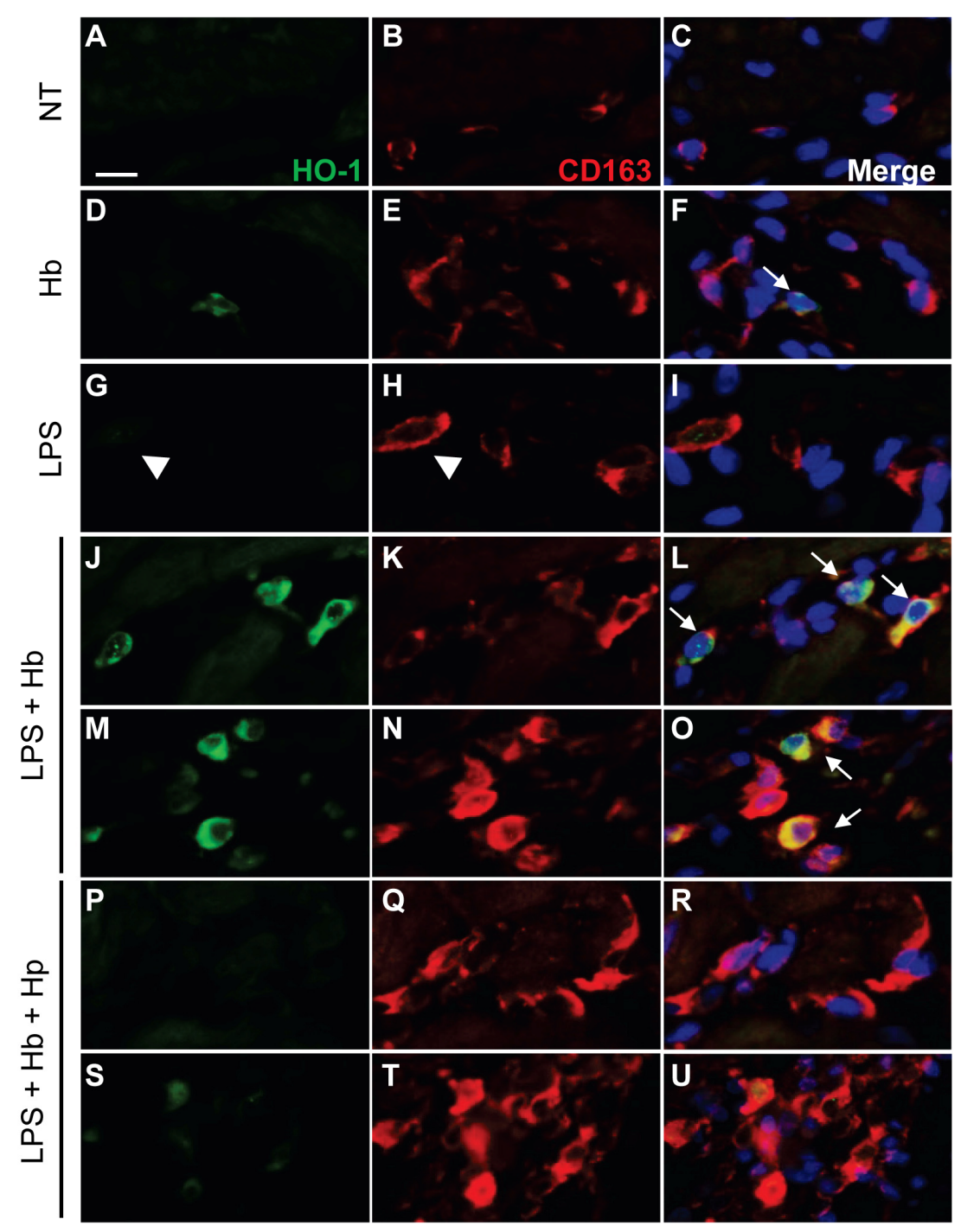

\subsection{Hp Reduces Myocardial Myeloperoxidase Expression Induced by LPS plus Hb}

We next examined the myocardial expression of myeloperoxidase (MPO), an enzyme found primarily in neutrophils and macrophages that is considered a reliable marker of inflammation and oxidative stress [20]. Immunofluorescence analysis of MPO showed minimal staining in surgical controls and $\mathrm{Hb}$ infused animals (Figure 4A,B). $\mathrm{MPO}^{+}$cells were detected sporadically in large myocardial blood vessels of endotoxemic animals (Figure 4C). Animals infused with LPS $+\mathrm{Hb}$ showed a higher incidence of $\mathrm{MPO}^{+}$infiltrates primarily in the lumen and subendothelial layers of 
blood vessels located in the ventricular myocardium (Figure 4D,E). Co-administration of Hp decreased the number of detectable $\mathrm{MPO}^{+}$cells (Figure 4F). Western blot analysis of myocardial tissue extracts from animals infused with LPS or $\mathrm{Hb}$ alone showed no significant change in MPO expression compared to surgical controls (Figure 4G). In contrast, LPS + Hb induced a significant increase in MPO expression that was prevented with the co-administration of $\mathrm{Hp}$.

Figure 4. Immunofluorescence analysis of myocardial MPO expression in surgical control animals (A) and animals infused with $\mathrm{Hb}$ (B), LPS (C), LPS $+\mathrm{Hb}$ (D, E) or $\mathrm{LPS}+\mathrm{Hb}+\mathrm{Hp}(\mathbf{F}) . \mathrm{MPO}^{+}$infiltrates expressed red-stained azurophilic granules in the lumen (D) and subendothelial layers (E) of myocardial blood vessels. Asterisks in Panel D and $\mathrm{E}$ denote the lumen of a blood vessel. Nuclei were counterstained with Hoechst 33342 (blue). Scale bar $=20 \mu \mathrm{m}(\mathrm{A}-\mathrm{D}, \mathrm{F})$ or $10 \mu \mathrm{m}(\mathrm{E})$. (G) Myocardial MPO expression by Western blot. Representative blots for MPO and tubulin are shown. Densitometry values as a ratio to tubulin were normalized to NT and presented as the means \pm SEM. ${ }^{*} p<0.05 v s$. NT, $\# p<0.05 v s$. LPS $+\mathrm{Hb}(n=4$ animals per group $)$.
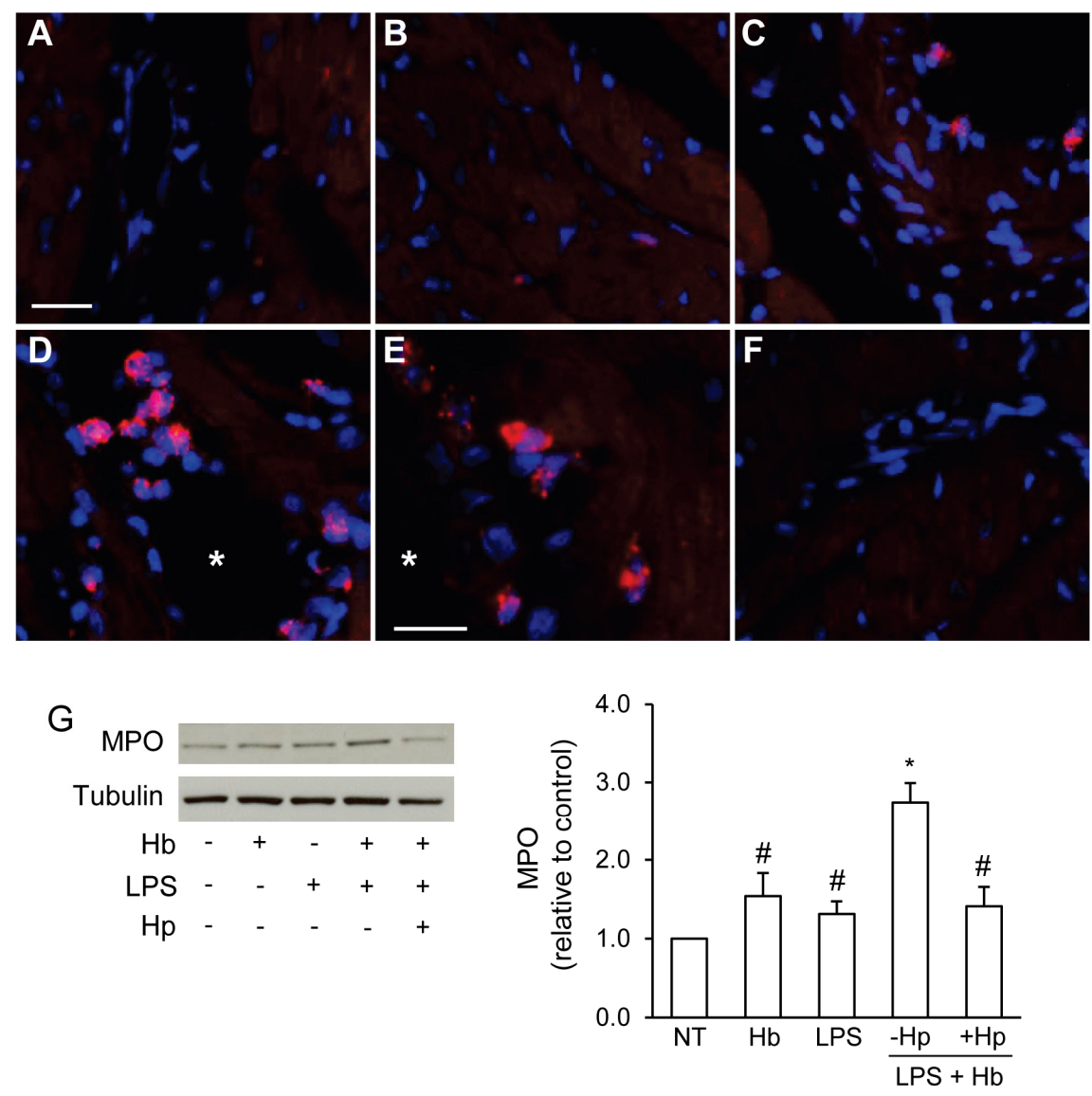

\subsection{Hp Protects against Myocardial Oxidative DNA Damage and Apoptosis Induced by LPS plus Hb}

To further investigate oxidative stress and injury in this model, we analyzed 8-hydroxy-2'-deoxyguanosine $(8-\mathrm{OHdG})$, a marker of oxidative DNA damage. Minimal 8-OHdG staining was detected in surgical controls or in animals infused with LPS or Hb alone (Figure 5A). Increased 8-OHdG reactivity was observed in animals infused with LPS $+\mathrm{Hb}$, particularly in the 
endothelial lining of many medium- to small-sized vessels in the ventricular regions of the myocardium. In LPS $+\mathrm{Hb}$, but not LPS alone, perivascular macrophages and infiltrates also showed increased $8-\mathrm{OHdG}$ reactivity. An increase in $8-\mathrm{OHdG}$ reactivity was also evident in myocytes located in the vicinity of 8-OHdG positive blood vessels. Hp co-administration markedly reduced the extent of $8-\mathrm{OHdG}$ staining in cardiac blood vessels and within the myocardium (Figure 5A).

Figure 5. (A) Immunofluorescence analysis of 8-hydroxy-2'-deoxyguanosine (8-OHdG) in myocardial sections of surgical control animals (NT), LPS, Hb, LPS $+\mathrm{Hb}$ and $\mathrm{LPS}+\mathrm{Hb}+\mathrm{Hp}$. Pre-incubation of primary antibody with 8-OHdG (adsorption control) completely blocked the positive staining associated with the same blood vessel depicted in the LPS + Hb panel. (B) Representative images of TUNEL staining (red) and Hoechst-stained nuclei are shown. Quantification of TUNEL staining (total positive cells and late apoptotic cells) was performed as described in the Experimental Section. Means \pm SEM for each group are shown. ${ }^{*} p<0.05 v s . \mathrm{NT},{ }^{* *} p<0.05 v s$. LPS + Hb. Scale bar $=50 \mu \mathrm{m}$.
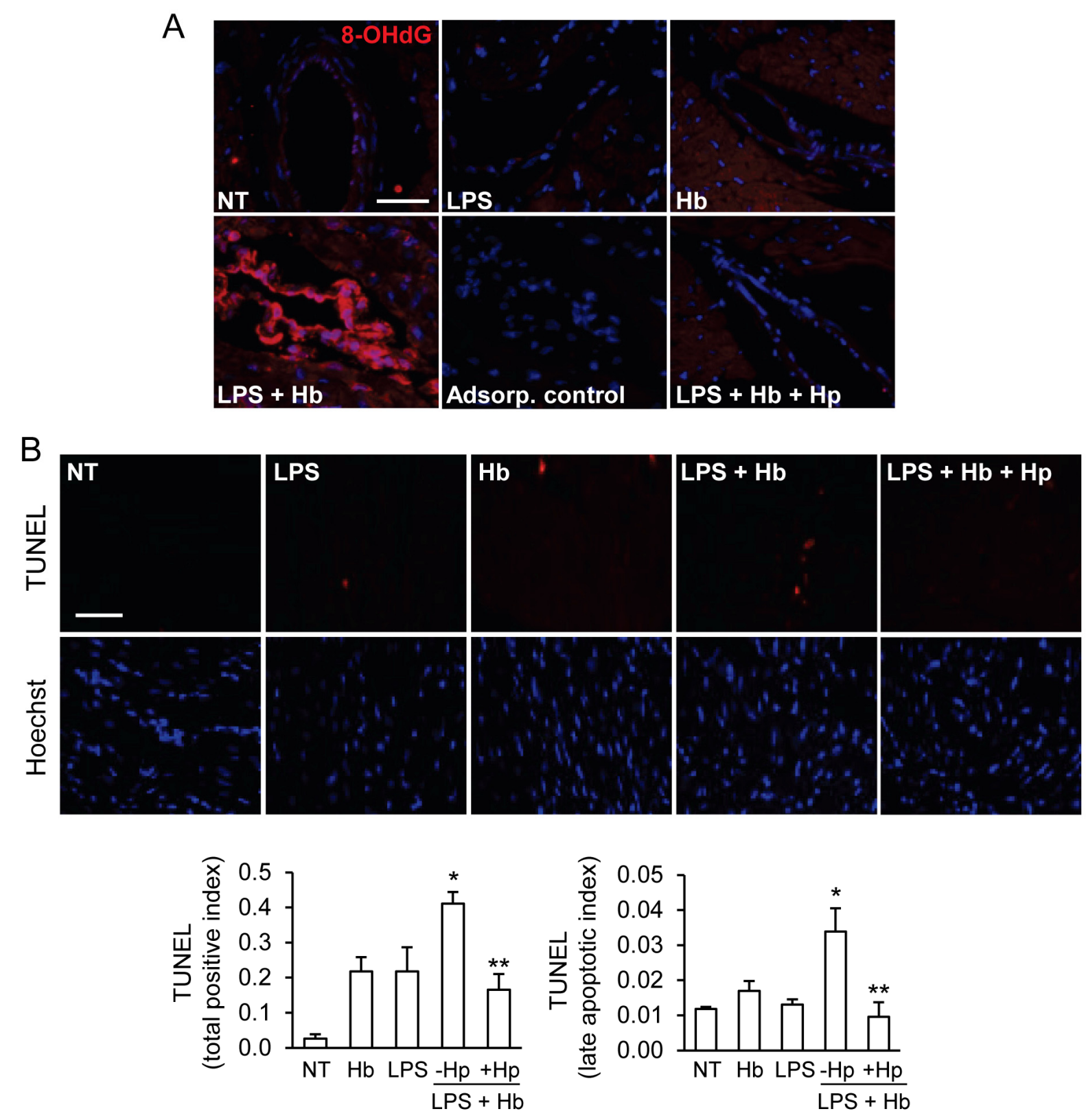
To further evaluate DNA damage and apoptosis, myocardial sections were analyzed by the TUNEL assay (Figure 5B). Two types of TUNEL positive nuclei were distinguishable and quantified separately in these sections. The total positive cell index represented the nuclei with detectable TUNEL reactivity that showed no discernible changes in nuclear morphology. The late apoptotic (or necrotic) index represented the TUNEL-positive nuclei with confirmed nuclear condensation, disintegration or apoptotic body formation. In general, TUNEL-positive nuclei were evident among both myocytes, and non-myocyte (i.e., perivascular macrophages, endothelial cells) populations though the proportion of TUNEL-positive cells with a normal nuclear appearance were significantly greater than the confirmed late apoptotic (necrotic) cells. Infusion with LPS or $\mathrm{Hb}$ alone did not increase the total positive or late apoptotic cells compared to surgical controls. In contrast, LPS + Hb increased TUNEL reactivity among both the total positive cells and late apoptotic cells (Figure 5B). Hp administration significantly reduced TUNEL positivity induced by LPS $+\mathrm{Hb}$.

\section{Discussion}

Studies in animal models of endotoxemia have reported that extracellular $\mathrm{Hb}$ exposure significantly increases lethality and may be associated with cardiovascular dysfunction $[5,6]$. The synergism between $\mathrm{Hb}$ and LPS is often attributed to $\mathrm{Hb}$ binding to LPS, causing disaggregation of LPS multimers and enhancing the exposure to LPS lipid A moieties [21,22]. Previous studies have also suggested that $\mathrm{Hb}$ augments LPS-induced TNF-alpha response in cultured monocytes and in vivo, an effect thought to contribute to the synergistic toxicity of $\mathrm{Hb}$ and LPS [23,24]. Enzymes, such as xanthine oxidase, phagocyte NADPH oxidase and MPO, have also been implicated in the overproduction of reactive oxygen species in sepsis and endotoxemia [25-29]. Several studies have investigated the role of MPO in monitoring cardiovascular disease primarily related to the inflammation observed in atherosclerosis [20], while the DNA oxidative product, 8-OHdG, is reported to be a relevant marker of sepsis-related oxidative stress and outcome [30]. In the context of the present study, we postulate that the oxidative microenvironment created by activated circulating inflammatory cells or tissue resident cells along the endothelial lining and within perivascular sites could be a major factor driving the oxidative reactions of $\mathrm{Hb}$ or its breakdown products [31]. Consistent with this view, the enhanced redox activity of various Hbs was shown to dramatically enhance LPS-induced apoptosis in endothelial cell culture [32]. Endothelial dysfunction is a hallmark of sepsis, which is often characterized by increased vascular permeability induced by LPS-mediated processes in several tissues, including the right and left ventricular coronary micro-vasculature [33,34]. Thus, LPS-induced loss of barrier function may represent a contributing mechanism underlying the extravasation of extracellular $\mathrm{Hb}$ or its breakdown products into perivascular sites.

Consistent with these concepts, the present findings suggest that extracellular $\mathrm{Hb}$ exposure in endotoxemic guinea pigs can exacerbate inflammation, oxidative stress and cellular injury. These events are likely mediated by increased vascular permeability and perivascular myocardial exposure to free $\mathrm{Hb}$ or its breakdown products. We show that $\mathrm{Hb}$ infusion following LPS administration, but not either insult alone, increased myocardial iron deposition and HO-1 expression supporting the extravascular exposure to $\mathrm{Hb}$ with subsequent heme release. Enhanced MPO- and 8-OHdG-positive infiltrates, as well as 8-OHdG-positive perivascular and endothelial cells 
of myocardial blood vessels are supportive of increased inflammation and oxidative stress. This is also consistent with the increased TUNEL reactivity observed in myocyte and non-myocyte populations.

$\mathrm{Hp}$ is an important acute phase protein that initially increases in response to inflammation and infection. Other $\mathrm{Hb}$ detoxification proteins within this class also include hemopexin and ferritin, which participate in heme clearance and intracellular iron storage, respectively. Additional acute phase reactants relevant to infection and endotoxemia include: C-reactive protein, clotting factors, hepcidin and complement that participates in bacterial opsonization [35]. Our results demonstrate that the co-administration of $\mathrm{Hp}$ blocked the myocardial events induced by the combination of LPS and $\mathrm{Hb}$. Several mechanisms may explain the protective activity of Hp. First, Hp may limit the translocation of $\mathrm{Hb}$ across the hyperpermeable endothelium. Second, Hp may prevent the release of heme into the myocardium and the vasculature, and third, $\mathrm{Hp}$ may control the redox activity of $\mathrm{Hb}$ driven by inflammation-derived hydro- and lipo-peroxides [36,37]. Together, the present data support a potentially useful therapeutic role for $\mathrm{Hp}$ in clinical or disease settings associated with the increased co-exposure to $\mathrm{Hb}$ and LPS, such as sepsis with concomitant trauma, surgery or blood transfusions.

\section{Experimental Section}

\subsection{Reagents and Antibodies}

Stroma-free human $\mathrm{Hb}$ was purified from hemolysate to remove catalase by ion exchange chromatography on a diethylaminoethanol (DEAE) Sephadex column (GE-Healthcare, Sweden) and concentrated to $150 \mathrm{mg} / \mathrm{mL}$ in normal saline. Human multimeric phenotype specified product, absent dimeric Hp, was provided by Benesis Corporation (Osaka, Japan) at a concentration of $20 \mathrm{mg} / \mathrm{mL}$ in normal saline. Hp was then concentrated to $60 \mathrm{mg} / \mathrm{mL}$. Standards of Hb-Hp complex for analytical size exclusion chromatography were separated from excessive (non-bound) $\mathrm{Hb}$ by gel chromatography on a HiLoad 26/60 Superdex 200 chromatography column (GE Healthcare Life Sciences, Piscataway, NJ, USA) and buffer exchanged into $50 \mathrm{mM}$ phosphate-buffered saline (PBS). Rabbit polyclonal antibody to HO-1 was purchased from Enzo Life Sciences (Farmingdale, NY, USA). Mouse anti-CD163 clone EDHu-1 was obtained from AbD Serotec (Raleigh, NC, USA). Rabbit polyclonal anti-tubulin was obtained from Santa Cruz Biotechnologies (Santa Cruz, CA, USA). Rabbit polyclonal antibody to MPO was purchased from Cell Signaling Technology (Danvers, MA, USA). Rabbit monoclonal antibody to MPO was obtained from Abcam (Cambridge, MA, USA). Mouse monoclonal antibody to 8-OHdG (clone N45.1) was purchased from Oxis International (Foster City, CA, USA).

\subsection{Animal Experimental Protocol}

Male Hartley guinea pigs (Charles Rivers Laboratories, Wilmington, MA, USA) were acclimated for one week upon arrival to the Food and Drug Administration (FDA) Center for Biologics Evaluation and Research (CBER) animal care facility. All animals weighed 400-450 g before surgery. Animal protocols were approved by the FDA CBER Institutional Animal Care and Use Committee, with all experimental procedures performed in adherence to the National Institutes of Health guidelines on the use of experimental animals. Surgical preparation was performed as previously described for implantation of indwelling venous and arterial catheters. After a $24 \mathrm{~h}$ period of post-surgical recovery, 
animals were randomized to one of five groups as follows: (1) surgical non-treated control; (2) LPS (10 mg/kg, i.v.); (3) Hb (150 mg, i.v.); (4) LPS + Hb; and (5) LPS + Hb + Hp (150 mg Hb + $180 \mathrm{mg}$ $\mathrm{Hp}$, to ensure complete binding of $\mathrm{Hb}$ ). All groups received equal infusion volumes normalized with $0.9 \% \mathrm{NaCl}$. LPS was injected via an indwelling jugular catheter $2 \mathrm{~h}$ prior to administration of saline control, $\mathrm{Hb}$ or $\mathrm{Hb}+\mathrm{Hp}$. This LPS challenge produced sublethal endotoxemia allowing for the evaluation of $\mathrm{Hb}$ and $\mathrm{Hb}+\mathrm{Hp}$ effects in the absence of mortality. In the three $\mathrm{Hb}$ infusion groups, the maximal plasma levels of $\mathrm{Hb}$ measured as heme equivalents were approximately $150 \mu \mathrm{M}(260 \mathrm{mg} / \mathrm{dL} \mathrm{Hb})$.

Blood $(0.2 \mathrm{~mL})$ was collected at baseline and after administration of control or test solution as follows: $\mathrm{T}=0$ (end administration), $0.5,1,2,4,6,12$ and $24 \mathrm{~h}$ (study termination). Urine was collected over the initial $4 \mathrm{~h}$ following administration of test or control solutions and at $24 \mathrm{~h}$ (study termination). Tissue harvesting was performed at $24 \mathrm{~h}$ following intraperitoneal administration of Euthasol $(0.22 \mathrm{mg} / \mathrm{kg})$ to induce deep anesthesia. Whole body perfusion was performed with $60 \mathrm{~mL}$ of cold $0.9 \% \mathrm{NaCl}$ to remove blood from major organ systems. Heart was cut into two longitudinal sections starting at the apex through the septum to the aortic root to expose the right and left ventricular walls and the septum. Similarly, right and left kidneys were cut into two longitudinal sections to expose the cortex and medulla. Half of the tissue was fixed in formalin, and half was snap frozen in liquid nitrogen.

\subsection{Plasma Hemoglobin Distribution}

Following administration, $\mathrm{Hp}$ bound and unbound fractions of $\mathrm{Hb}$ were determined by size exclusion high performance liquid chromatography (SEC-HPLC) using a Waters 600 controller, a Waters 600 pump and a Waters 2499 photodiode array detector (Waters, Corp, Millford, MA, USA). Plasma samples were separated on a BioSep-SEC-S3000 $(600 \times 7.5 \mathrm{~mm})$ column (Phenomenex, Torrance, CA, USA) with $50 \mathrm{mM}$ potassium phosphate, $\mathrm{pH} 7.4$ as the mobile phase. Column injections were maintained at consistent volumes using a $50-\mu \mathrm{L}$ injection loop. All samples were monitored at $\lambda=280 \mathrm{~nm}$ and $\lambda=405 \mathrm{~nm}$ in dual wavelength mode. Hb bound and unbound in plasma was determined by dividing the $\mathrm{Hb}$ peak area and the $\mathrm{Hb}-\mathrm{Hp}$ peak area by additive areas under the $\mathrm{Hb}-\mathrm{Hp}$ chromatographic peak (13-17 min elution) and the Hb chromatographic peak (21 min elution) at $\lambda=405 \mathrm{~nm}$.

\subsection{Non-heme Iron Immunohistochemistry}

Hearts and kidneys were fixed in $10 \%$ formalin for $24 \mathrm{~h}$, then stored in $100 \%$ isopropanol, embedded in paraffin, and $5 \mu \mathrm{m}$ sections were prepared. Non-heme ferric iron deposition was detected using Perls' method with DAB intensification. Sections were dewaxed in Safe Clear ${ }^{\mathrm{TM}}$ (Houston, TX, USA) and rehydrated in graded ethanol and deionized water. Tissues were then incubated with Perls' iron reagent containing 5\% potassium ferrocyanide and $2 \%$ hydrochloric acid for 45 min at room temperature and rinsed in deionized water. Sections were then incubated with $0.3 \%$ hydrogen peroxide and $0.01 \mathrm{M}$ sodium azide in methanol for $30 \mathrm{~min}$ at room temperature. All sections were then rinsed in $0.1 \mathrm{M}$ phosphate buffer, $\mathrm{pH} 7.4$, incubated with $\mathrm{DAB}$ for three minutes, washed in deionized water and lightly counterstained with Gill's II hematoxylin. After dehydrating in graded ethanol and 
Safeclear, slides were mounted in Permount and coverslipped. Images were obtained using an Olympus IX71 inverted microscope.

\subsection{Preparation of Heart Tissue Lysates}

Frozen heart samples from the ventricular compartment were dounce-homogenized in the presence of ice-cold modified radioimmunoprecipitation assay (RIPA) buffer (50 mM Tris, $150 \mathrm{mM}$ sodium chloride, $1 \%$ IgePal $^{\circledR}-630,0.5 \%$ sodium deoxycholate, $1 \mathrm{mM}$ ethylenediaminetetraacetic acid (EDTA) containing protease inhibitors (Cocktail Set III, Calbiochem, CA, USA). Lysates were centrifuged at $10,000 \mathrm{rpm}$ at $4{ }^{\circ} \mathrm{C}$ for $30 \mathrm{~min}$. Supernatants were collected and stored at $-80{ }^{\circ} \mathrm{C}$. Protein concentrations were measured using the bicinchoninic acid (BCA) protein assay (Thermo Scientific, Rockford, IL, USA).

\subsection{Western Blot Analyses}

Heart lysates were resolved on $4 \%-12 \%$ Bis-Tris gels, transferred to polyvinylidene fluoride (PVDF) membranes and blocked for $1 \mathrm{~h}$ in Tris buffered saline with $0.1 \%$ Tween-20 (TBS-T) and 5\% nonfat dry milk. Membranes were incubated overnight at $4{ }^{\circ} \mathrm{C}$ with antibodies to HO-1 (1:2500) and MPO (1:2000) in TBS-T with $1 \%$ nonfat dry milk or $3 \%$ bovine serum albumin (for MPO), washed and then incubated with a relevant horseradish peroxidase (HRP)-conjugated secondary antibody for 1 $\mathrm{h}$. The signal was developed using the ECL Plus chemiluminescence kit (Amersham, Arlington Heights, IL) and detected on HyperECL film. Densitometry analysis was performed using ImageJ software (National Institutes of Health, Bethesda, MD, USA) with normalization to tubulin.

\subsection{Immunofluorescence}

Paraffin-embedded slides were dewaxed and rehydrated. Slides were heat-treated in a microwave oven for $15 \mathrm{~min}$ in $10 \mathrm{mM}$ sodium citrate buffer, $\mathrm{pH} \mathrm{6.0,} \mathrm{cooled} \mathrm{for} 30 \mathrm{~min}$ at room temperature and rinsed with deionized water and PBS-T. Sections were then blocked in PBS-T with 5\% goat or horse serum for $1 \mathrm{~h}$ at room temperature followed by overnight incubation at $4{ }^{\circ} \mathrm{C}$ with antibodies to HO-1, MPO, CD163 and 8-OHdG. Sections were rinsed and incubated with relevant Alexa-Fluor 488- and Alexa-Fluor 555-conjugated secondary antibodies for $1 \mathrm{~h}$ at room temperature. Nuclei were counterstained with Hoechst 33342. For double labeling experiments, primary antibodies were mixed together and incubated overnight at $4{ }^{\circ} \mathrm{C}$. Sections stained with secondary antibodies alone showed no specific staining. Images were processed using an Olympus IX71 inverted microscope equipped with an Olympus DP70 digital camera.

\subsection{TUNEL Assay}

Hearts were removed and fixed in neutral buffered 10\% formalin at room temperature for $24 \mathrm{~h}$ before embedding in paraffin and sectioning. Sections were deparaffinized, antigen retrieved with proteinase $\mathrm{K}$ treatment ( $20 \mu \mathrm{g} / \mathrm{mL}$ for $20 \mathrm{~min}$ at room temperature) and then subjected to TUNEL (terminal deoxynucleotidyl transferase-mediated dUTP nick end labeling) staining according to the manufacturer's instructions (Apotag Red In Situ Apoptosis Detection kit, Millipore, Billerica, MA, 
USA). Negative controls performed in the absence of the terminal deoxynucleotidyl transferase TdT enzyme showed no TUNEL reactivity. The TUNEL-positive nuclei and the total number of nuclei were counted in 6 fields of view per slide captured at 400× magnification. The average number of nuclei counted per field for each group was as follows; non-treated controls (175 \pm 4$), \mathrm{Hb}(170 \pm 13)$, LPS $(162 \pm 17), \mathrm{LPS}+\mathrm{Hb}(179 \pm 7)$ and LPS $+\mathrm{Hb}+\mathrm{Hp}(188 \pm 10)$. The TUNEL-positive cell index was calculated as the number of nuclei with detectable TUNEL reactivity regardless of staining intensity or nuclear morphology divided by the total number of nuclei. The late apoptotic index was calculated as the number of TUNEL-positive nuclei with confirmed nuclear condensation or disintegration divided by the total number of nuclei.

\subsection{Statistical Analysis}

Data are represented as the means \pm SEM for replicate experiments. Statistical analysis was performed by one-way ANOVA with a post-hoc Bonferroni's test for all normally distributed, equal variance and equal number comparisons. A non-parametric one-way ANOVA was performed for comparisons with unequal distributions and non-matched group sizes. All analyses were performed using GraphPad Prism version 5 software. A $p$-value $<0.05$ was considered statistically significant.

\section{Conclusions}

Extracellular $\mathrm{Hb}$ enhances the markers of iron deposition, inflammation, oxidative stress, and cell death in the myocardium of endotoxemic guinea pigs. The co-administration of $\mathrm{Hp}, \mathrm{a} \mathrm{Hb}$ scavenger protein, attenuated the myocardial events observed. These findings suggest that the synergistic toxicity induced by the co-exposure to LPS and $\mathrm{Hb}$ may be counteracted by therapeutic approaches designed to prevent or eliminate the accumulation of free $\mathrm{Hb}$. This may have important implications for the management of sepsis and/or septic shock with concomitant trauma, surgery, or blood transfusions.

\section{Acknowledgments}

This project was supported in part by an appointment of Xiaoyuan Zhang to a Research Fellowship Program for the Center for Biologics Evaluation and Research, administered by the Oak Ridge Institute for Science and Education through an interagency agreement between the U.S. Department of Energy and the U.S. Food and Drug Administration, and FDA Modernizing Science Grants awarded to Paul W. Buehler and Felice D'Agnillo.

\section{Author Contributions}

Paul W. Buehler and Felice D'Agnillo conceived the study, wrote the paper, and prepared the figures. Jin Hyen Baek, Xiaoyuan Zhang, Matthew C. Williams, Paul W. Buehler, and Felice D'Agnillo carried out experiments and performed data collection and analysis. Dominik J. Schaer provided materials and edited the paper. All authors have read, edited, and approved the final version of the paper.

\section{Conflicts of Interest}

The authors declare no conflict of interest. 


\section{References}

1. Zanotti-Cavazzoni, S.L.; Hollenberg, S.M. Cardiac dysfunction in severe sepsis and septic shock. Curr. Opin. Crit Care 2009, 15, 392-397.

2. Flynn, A.; Chokkalingam Mani, B.; Mather, P.J. Sepsis-induced cardiomyopathy: A review of pathophysiologic mechanisms. Heart Fail Rev. 2010, 15, 605-611.

3. Balija, T.M.; Lowry, S.F. Lipopolysaccharide and sepsis-associated myocardial dysfunction. Curr. Opin. Infect. Dis. 2011, 24, 248-253.

4. Suliman, H.B.; Welty-Wolf, K.E.; Carraway, M.; Tatro, L.; Piantadosi, C.A. Lipopolysaccharide induces oxidative cardiac mitochondrial damage and biogenesis. Cardiovasc. Res. 2004, 64, 279-288.

5. Su, D.; Roth, R.I.; Yoshida, M.; Levin, J. Hemoglobin increases mortality from bacterial endotoxin. Infect. Immun. 1997, 65, 1258-1266.

6. Krishnamurti, C.; Carter, A.J.; Maglasang, P.; Hess, J.R.; Cutting, M.A.; Alving, B.M. Cardiovascular toxicity of human cross-linked hemoglobin in a rabbit endotoxemia model. Crit. Care Med. 1997, 25, 1874-1880.

7. McGahan, M.C.; Grimes, A.M.; Fleisher, L.N. Hemoglobin exacerbates the ocular inflammatory response to endotoxin. Graefes Arch. Clin. Exp. Ophthalmol. 1996, 234, 643-647.

8. Larsen, R.; Gozzelino, R.; Jeney, V.; Tokaji, L.; Bozza, F.A.; Japiassu, A.M.; Bonaparte, D.; Cavalcante, M.M.; Chora, A.; Ferreira, A.; et al. A central role for free heme in the pathogenesis of severe sepsis. Sci. Transl. Med. 2010, 2, doi:10.1126/scitranslmed.3001118.

9. Adamzik, M.; Hamburger, T.; Petrat, F.; Peters, J.; de Groot, H.; Hartmann, M. Free hemoglobin concentration in severe sepsis: Methods of measurement and prediction of outcome. Crit. Care 2012, 16, R125.

10. Janz, D.R.; Bastarache, J.A.; Peterson, J.F.; Sills, G.; Wickersham, N.; May, A.K.; Roberts, L.J., 2nd; Ware, L.B. Association between cell-free hemoglobin, acetaminophen, and mortality in patients with sepsis: An observational study. Crit. Care Med. 2013, 41, 784-790.

11. Janz, D.R.; Bastarache, J.A.; Sills, G.; Wickersham, N.; May, A.K.; Bernard, G.R.; Ware, L.B. Association between haptoglobin, hemopexin and mortality in adults with sepsis. Crit. Care 2013, 17, R272.

12. Smithies, O.; Connell, G.E.; Dixon, G.H. Inheritance of haptoglobin subtypes. Am. J. Hum. Genet. 1962, 14, 14-21.

13. Nosslin, B.F.; Nyman, M. Haptoglobin determination in diagnosis of haemolytic diseases. Lancet 1958, 1, 1000-1001.

14. Andersen, C.B.; Torvund-Jensen, M.; Nielsen, M.J.; de Oliveira, C.L.; Hersleth, H.P.; Andersen, N.H.; Pedersen, J.S.; Andersen, G.R.; Moestrup, S.K. Structure of the haptoglobin-haemoglobin complex. Nature 2012, 489, 456-459.

15. Connell, G.E.; Dixon, G.H.; Smithies, O. Subdivision of the three common haptoglobin types based on "hidden" differences. Nature 1962, 193, 505-506. 
16. Boretti, F.S.; Buehler, P.W.; D’Agnillo, F.; Kluge, K.; Glaus, T.; Butt, O.I.; Jia, Y.; Goede, J.; Pereira, C.P.; Maggiorini, M.; et al. Sequestration of extracellular hemoglobin within a haptoglobin complex decreases its hypertensive and oxidative effects in dogs and guinea pigs. J. Clin. Invest 2009, 119, 2271-2280.

17. Lim, S.K.; Kim, H.; Lim, S.K.; bin Ali, A.; Lim, Y.K.; Wang, Y.; Chong, S.M.; Costantini, F.; Baumman, H. Increased susceptibility in Hp knockout mice during acute hemolysis. Blood 1998, 92, 1870-1877.

18. Hashimoto, K.; Nomura, K.; Nakano, M.; Sasaki, T.; Kurosawa, H. Pharmacological intervention for renal protection during cardiopulmonary bypass. Heart Vessel. 1993, 8, 203-210.

19. Bunn, H.F.; Jandl, J.H. The renal handling of hemoglobin. II. Catabolism. J. Exp. Med. 1969, 129, 925-934.

20. Schindhelm, R.K.; van der Zwan, L.P.; Teerlink, T.; Scheffer, P.G. Myeloperoxidase: A useful biomarker for cardiovascular disease risk stratification? Clin. Chem. 2009, 55, 1462-1470.

21. Kaca, W.; Roth, R.I.; Levin, J. Hemoglobin, a newly recognized lipopolysaccharide (LPS)-binding protein that enhances LPS biological activity. J. Biol. Chem. 1994, 269, 25078-25084.

22. Bahl, N.; Du, R.; Winarsih, I.; Ho, B.; Tucker-Kellogg, L.; Tidor, B.; Ding, D.L. Delineation of lipopolysaccharide (LPS)-binding sites on hemoglobin: From in silico predictions to biophysical characterization. J. Biol. Chem. 2011, 286, 37793-37803.

23. Bodet, C.; Chandad, F.; Grenier, D. Hemoglobin and LPS act in synergy to amplify the inflammatory response. J. Dent. Res. 2007, 86, 878-882.

24. Gorczynski, R.M.; Alexander, C.; Bessler, W.; Fournier, K.; Hoffmann, P.; Mach, J.P.; Manuel, J.; Ramakrishna, V.; Rietschel, E.T.; Song, L.; et al. Characterization of an interaction between fetal hemoglobin and lipid A of LPS resulting in augmented induction of cytokine production in vivo and in vitro. Int. Immunopharmacol. 2004, 4, 1859-1872.

25. Luchtemberg, M.N.; Petronilho, F.; Constantino, L.; Gelain, D.P.; Andrades, M.; Ritter, C.; Moreira, J.C.; Streck, E.L.; Dal-Pizzol, F. Xanthine oxidase activity in patients with sepsis. Clin. Biochem. 2008, 41, 1186-1190.

26. Wu, J.; Xu, H.; Yang, M.; Martin, C.M.; Kvietys, P.R.; Rui, T. NADPH oxidase contributes to conversion of cardiac myocytes to a proinflammatory phenotype in sepsis. Free Radic. Biol. Med. 2009, 46, 1338-1345.

27. Kothari, N.; Keshari, R.S.; Bogra, J.; Kohli, M.; Abbas, H.; Malik, A.; Dikshit, M.; Barthwal, M.K. Increased myeloperoxidase enzyme activity in plasma is an indicator of inflammation and onset of sepsis. J. Crit. Care 2011, 26, 435.e1-435.e7.

28. Gao, M.; Ha, T.; Zhang, X.; Liu, L.; Wang, X.; Kelley, J.; Singh, K.; Kao, R.; Gao, X.; Williams, D.; et al. Toll-like receptor 3 plays a central role in cardiac dysfunction during polymicrobial sepsis. Crit. Care Med. 2012, 40, 2390-2399.

29. Huet, O.; Dupic, L.; Harrois, A.; Duranteau, J. Oxidative stress and endothelial dysfunction during sepsis. Front. Biosci. 2011, 16, 1986-1995.

30. Cheng, W.E.; Shih, C.M.; Hang, L.W.; Wu, K.Y.; Yang, H.L.; Hsu, W.H.; Hsia, T.C. Urinary biomarker of oxidative stress correlating with outcome in critically septic patients. Intensive Care Med. 2007, 33, 1187-1190. 
31. Buehler, P.W.; D'Agnillo, F. Toxicological consequences of extracellular hemoglobin: biochemical and physiological perspectives. Antioxid. Redox. Signal. 2010, 12, 275-291.

32. D'Agnillo, F. Redox active hemoglobin enhances lipopolysaccharide-induced injury to cultured bovine endothelial cells. Am. J. Physiol. Heart Circ. Physiol. 2004, 287, H1875-H1882.

33. Langheinrich, A.C.; Ritman, E.L. Quantitative imaging of microvascular permeability in a rat model of lipopolysaccharide-induced sepsis: Evaluation using cryostatic micro-computed tomography. Invest Radiol. 2006, 41, 645-650.

34. Darwish, I.; Liles, W.C. Emerging therapeutic strategies to prevent infection-related microvascular endothelial activation and dysfunction. Virulence 2013, 4, 572-582.

35. Sankar, V.; Webster, N.R. Clinical application of sepsis biomarkers. J. Anesth. 2013, 27, 269-283.

36. Banerjee, S.; Jia, Y.; Siburt, C.J.; Abraham, B.; Wood, F.; Bonaventura, C.; Henkens, R.; Crumbliss, A.L.; Alayash, A.I. Haptoglobin alters oxygenation and oxidation of hemoglobin and decreases propagation of peroxide-induced oxidative reactions. Free Radic. Biol. Med. 2013, 53, 1317-1326.

37. Buehler, P.W.; Abraham, B.; Vallelian, F.; Linnemayr, C.; Pereira, C.P.; Cipollo, J.F.; Jia, Y.; Mikolajczyk, M.; Boretti, F.S.; Schoedon, G.; et al. Haptoglobin preserves the CD163 hemoglobin scavenger pathway by shielding hemoglobin from peroxidative modification. Blood 2009, 113, 2578-2586.

(C) 2014 by the authors; licensee MDPI, Basel, Switzerland. This article is an open access article distributed under the terms and conditions of the Creative Commons Attribution license (http://creativecommons.org/licenses/by/3.0/). 